\title{
The stem anatomy of the Clematis species (Ranunculaceae) in Taiwan
}

Sheng-Zehn Yang ( $\nabla$ yangsz@mail.npust.edu.tw)

National Pingtung University of Science and Technology https://orcid.org/0000-0001-8648-7507

Po-Hao Chen

National Pingtung University of Science and Technology

Chien-Fan Chen

Taiwan Forestry Research Institute

Original Article

Keywords: Ranunculaceae, ray indentation, ring bark, scale bark, vessel restriction, wedge phloem

Posted Date: April 2nd, 2021

DOI: https://doi.org/10.21203/rs.3.rs-351752/v1

License: (c) (i) This work is licensed under a Creative Commons Attribution 4.0 International License. Read Full License 


\section{Abstract \\ Background}

Studies on the stem anatomical characteristics of Taiwanese species from the Clematis genus (Ranunculaceae) are scarce. This study aimed to investigate and compare the patterns of secondary growth in stems of 22 Clematis species.

\section{Results}

The rhytidome is composed of periderm and non-conducting phloem and formed either cogwheel-like or continuous segment bark. Key features of the genus were stem with an irregular conformation, wedge-like phloem and rays, indentations in the axial parenchyma, ray dilatation, and narrow rays. Approximately eight Clematis species formed bark arc shape, which developed the cogwheel- like rhytidome. There were with approximately $27 \%$ of the Clematis species in Taiwan having 12 vascular bundles. The vessels dispersed throughout the stem were semi-ring-porous in most species but were ring-porous in others. No species had diffuse-porous vessels. The vessel restriction pattern was only found in the two shrubs, $C$. psilandra and $C$. tsugetorum. The primary xylem ring was located around the pith in $C$. uncinata var. uncinata, making its pith cavity hexagon in shape. Four species had the pith cavity feature. Narrow rays that occurred in the secondary xylem increased with increasing stem diameter.

\section{Conclusions}

The cambial variants described in this study provide a foundation for further morphological studies of the Clematis genus.

\section{Background}

The vascular cambium of climbing plants produces xylem and phloem under normal conditions, and both types of tissue have large amounts of parenchyma cells. These cells can be re-differentiated into meristematic cells, which may give rise to secondary vascular tissue, cork cambia, dilatation tissue, or adventitious buds (Mauseth 1988). Due to the uneven deposition of secondary xylem, stems are generally irregularly shaped after secondary growth (Carlquist 1991; Rajput et al. 2014) as diverse cambial variants, or secondary growth patterns are formed. The stems examined generally developed axial vascular elements in segments separating by the wider xylem and phloem rays, forming xylem in plate type. This type is derived from a single cambium, according to Angyalossy et al. (2012, 2015). Many families develop this variant, including Ranunculaceae (Carlquist 2001).

The Ranunculaceae family comprises approximately 60 genera and 2500 species, including approximately 300 Clematis species worldwide (Wang and Bartholomew 2007), of which 22 are found in Taiwan (Yang and Huang 1996). The life forms of Clematis species include shrubs, herbs, and perennial climbers (lianas). Within the Ranunculaceae, several cambial variants have been reported (Angyalossy et al. 2012, 2015).

Previous anatomical studies of Ranunculaceae have mainly concentrated on the genus Clematis (Smith 1928; Sieber and Kucera 1980; Gregory 1994; Carlquist 1995). Few secondary rays begin abruptly and occur in C. cirrbosa, C. flammula, C. montevidensis, $C$. vitalba, and $C$. viticella (Schweingruber et al. 2011) with increasing the stem diameter. Carlquist (1995) indicated that new rays are wide multiseriate from their origin, and are initiated abruptly rather than as narrow rays that gradually increase in width in Clematis genus. Beck (2011) described that closely spaced, narrow rays occurred in groups will simulate large rays which terms as aggregate rays. The secondary or new rays also occur in woody climbers in two families, Cucurbitaceae (Carlquist 1992) and Aristolochiaceae (Carlquist 1993). The cambium in the secondary vascular tissue forms increasing ray-like parenchyma in Clematis flammula var. maritima (Isnard et al. 2003a). Interfascicular cambia ray width increases with increasing stem diameter in C. alpina subsp. alpina, that the rays are wedge-like in shape (Schweingruber et al. 2011).

The phellogen of $C$. alpina and $C$. vitalba produces cork cells and forms the phellem (cork) layers is located outside the secondary phloem (Schweingruber et al. 2011). The complex tissue region of the periderm and enclosed non-conducting phloem is called the rhytidome (Beck, 2011), which is generally comprised of successive cork and non-conducting phloem. According to the arrangement and detachment degree of rhytidome, two types were formed: cogwheel-like rhytidome (ring bark) and continuous segment rhytidome (scale bark) (Esau 1958; Sieber and Kucera 1980; Evert 2006). C. vitalba is a vine with big and small vascular bundles (six each) in its primary state. It forms the pith cavity, its bark tissue develops an arc, and the stem appears in a cogwheel-like form due to the different activities in the fascicular and interfascicular regions (Sieber and Kucera 1980).

In C. vitalba, the cambium is dented towards the pith in the broad rays region (Sieber and Kucera 1980), and in C. alpina and C. pickerringii, the interfascicular areas always have indentations (Carlquist 1995). Owing to the presence of thin-walled axial parenchyma in latewood adjacent to the thin-walled ray cells, wedge-shaped indentations can be seen in the interfascicular region (Sieber and Kucera 1980; Carlquist 1995). However, the indentations are strands of thinwalled axial parenchyma near the broader rays. Isnard et al. (2003a, b) defined four developmental stages of $C$. flammula var. maritima, $C$. recta, and $C$. vitalba by the appearance of the periderms, phloem fibers, rhytidome, and wide rays. The axial parenchyma of $C$. alpina, $C$. columbiana, $C$. hirsutissima, and $C$. recta are paratracheal with semi-ring-porous wood and distinct annual rings, while $C$. flammula, $C$. vitalba, and $C$. viticella have ring-porous wood with annual rings (Schweingruber et al. 2011).

The xylem in plate variant was found in the genus Clematis (Carlquist 1995, Isnard et al. 2003b, Yang and Chen 2015), but the information about the patterns of secondary growth or cambial variants in Taiwan 22 Clematis species were scarce. As cambial variants constitute an extremely diverse morphology, the present study aimed to 1) provide detailed photographs of the features discussed and 2) provide a bracketed key based on the anatomical characteristics of the stems to facilitate the identification of irregular cambial activity in the Clematis genus in Taiwan.

Page 2/19 


\section{Methods}

\section{Research Materials}

Multiple samples of 22 Clematis species of the family Ranunculaceae were collected. The habits of these species included annual and perennial herbs, shrubs, and lianas growing in different forests. The dataset included species scientific name, collector, herbarium, and voucher number. The voucher species information of all Clematis species is presented in Table 1 . Approximately $48 \%$ of the species were endemic to Taiwan. Among them, Clematis psilandra and C. tsugetorum are shrubs growing at high elevations of approximately $2,300 \mathrm{~m}$ and $3,200 \mathrm{~m}$, respectively. The species $C$. pseudootophora is an herb rarely found in the field. The remaining 19 species are climbing vines in which $C$. montana grows at the highest elevation of approximately $3,200 \mathrm{~m}$. This species consists of only a few populations located at an elevation of 1,500-1,900 $\mathrm{m}$ in eastern Taiwan.

Table 1

Voucher specimens of the 22 Taiwanese Clematis species investigated

\begin{tabular}{|c|c|c|}
\hline Species & Collector & $\begin{array}{l}\text { Herbarium and } \\
\text { voucher number }\end{array}$ \\
\hline${ }^{*}$ Clematis akoensis Hayata & Po-Hao Chen & PPI75993 \\
\hline Clematis chinensis Osbeck var. chinensis & Po-Hao Chen & PPI73776 \\
\hline *Clematis chinensis Osbeck var. tatushanensis T.Y.A. Yang & Po-Hao Chen & PPI76140 \\
\hline Clematis crassifolia Benth. & Chien-Fan Chen & TAIF449023 \\
\hline${ }^{*}$ Clematis formosana Kuntze & Po-Hao Chen & PPI76748 \\
\hline *Clematis gouriana Roxb. ex DC. subsp. lishanensis T.Y.A. Yang \& T.C. Huang & Sheng-Zehn Yang & PPI57118 \\
\hline Clematis grata Wall. & Po-Hao Chen & PPI79191 \\
\hline Clematis henryi Oliv. var. henryi & Po-Hao Chen & PPI76097 \\
\hline *Clematis henryi Oliv. var. morii (Hayata) T.Y.A. Yang \& T.C. Huang & Her-Long Chiang & PPI65589 \\
\hline Clematis lasiandra Maxim. & Sheng-Zehn Yang & PPI57133 \\
\hline Clematis leschenaultiana DC. & Chien-Fan Chen & PPI63232 \\
\hline Clematis meyeniana Walp. & Chien-Fan Chen & PPI60238 \\
\hline Clematis montana Buch.-Ham. ex DC. & Chien-Fan Chen & PPI63771 \\
\hline *Clematis parviloba Gard. ex Champ. subsp. bartlettii (Yamam.) T.Y.A. Yang \& T.C. Huang & Guang-Pu Hsieh & PPI61169 \\
\hline Clematis pseudootophora M.Y. Fang & Chien-Fan Chen & TAIF455630 \\
\hline *Clematis psilandra Kitag. & Guang-Pu Hsieh & PPI60043 \\
\hline *Clematis tamurae T.Y.A. Yang \& T.C. Huang & Po-Hao Chen & PPI79723 \\
\hline Clematis tashiroi Maxim. & Po-Hao Chen & PPI78815 \\
\hline *Clematis terniflora DC. var. garanbiensis (Hayata) M.C. Chang & Po-Hao Chen & PPI78668 \\
\hline${ }^{*}$ Clematis tsugetorum Ohwi & Guang-Pu Hsieh & PPI68569 \\
\hline Clematis uncinata Champ. ex Benth. var. kinawensis (Ohwi) Ohwi & Kun-Ping Lo & PPI69701 \\
\hline Clematis uncinata Champ. ex Benth. var. uncinata & Jyuen-Jyie Chen & PPI63904 \\
\hline
\end{tabular}

\section{Research Methods}

Multiple samples of each species were collected, stems with thick bark and secondary growth characteristics were selected in the field. To keep the materials fresh and retain humidity, the stems were stored in a collecting bag. Different diameters of each plant were collected to compare various developmental stages and accurately assess the position of various vascular bundle tissues. One or three samples with obvious and easy-to-observe cambial variants were selected per species for photographs and the scoring of morphological characteristics. The morphological features of stem cross-sections in the investigated species were used to construct a comparison table.

In the laboratory, the fresh materials were cut into pieces approximately $5 \mathrm{~cm}$ long, and a freehand cross-section of each stem was made with a razor blade. The stem cross-section was immediately photographed using a Nikon D7100 SLR digital camera with a 1:1 lens (Lens AF Micro Nikon 60 mm 1:2.8D; Nikon Corporation, Tokyo, Japan). Cambial characteristics were measured and described. Quantitative anatomical traits, such as stem diameter, bark thickness, mean xylem width, and mean primary ray width, were determined using Image-J software (Ferreira and Rasband 2011). All specimens were oven-dried at $60^{\circ} \mathrm{C}$ 
for 4-5 days and then stored at $-20^{\circ} \mathrm{C}$ for $3-4$ days. They were then deposited in the Provincial Pingtung Institute (PPI) herbarium at the National Pingtung University of Science and Technology, Pingtung, Taiwan, for subsequent identification. The nomenclature follows the Flora of Taiwan volume II (Yang and Huang 1996). The materials of C. pseudootophora were permanently preserved in $75 \%$ aqueous alcohol because of its rarity.

The following stem features of each species were investigated (Table 2): locality; elevation (m); stem diameter (mm); phloem fibers (+/-); arc-like bark (+/-); cogwheel-like rhytidome (+/-); continuous segment rhytidome $(+/-)$; rhytidome layer number; rhytidome thickness (mm); wedge-like rays $(+/-)$; indentation of the axial parenchyma near the broader rays (+/-); restricted vessel pattern (+/-); ray dilatations (+/-); vascular bundle number; xylem width (mm) (mean \pm SD, $\mathrm{n}=$ xylem number); ray width $(\mu \mathrm{m})$ (mean $\pm \mathrm{SD}, \mathrm{n}=$ ray number); wide rays number; narrow rays number; ring-porous vessels (+/-); semi-ring-porous vessels (+/-); and developmental stage. The vessel restriction pattern, ray types, and axial parenchyma types were classified based on Carlquist (2001) and the IAWA Committee on Nomenclature (1964). Species were grouped based on the number of vascular bundles they contained (Smith 1928), with the 'central type' having 12 bundles, the 'many type' having $>12$ bundles, and the 'few type' having $<12$ bundles. 
Table 2

Morphological characteristics determined from stem cross-sections of Taiwanese Clematis species and each line for each sper

\begin{tabular}{|c|c|c|c|c|c|c|c|c|c|c|c|c|c|c|c|c|}
\hline $\begin{array}{l}\text { Characters } \\
\text { Scientific name }\end{array}$ & LO & $\begin{array}{l}\text { EL } \\
(m)\end{array}$ & $\begin{array}{l}\mathrm{SD} \\
(\mathrm{mm})\end{array}$ & PF & AR & COG & SE & $\begin{array}{l}\text { RHL } \\
\text { (no.) }\end{array}$ & $\begin{array}{l}\text { RHT } \\
(\mathrm{mm})\end{array}$ & WER & ID & VR & RD & $\begin{array}{l}\text { VB } \\
\text { (no.) }\end{array}$ & $\begin{array}{l}X W \\
(\mathrm{~mm})\end{array}$ & $\begin{array}{l}\text { RW } \\
(\mu \mathrm{m})\end{array}$ \\
\hline${ }^{*}$ C. akoensis & $\begin{array}{l}\text { Shouka, Pingtung } \\
\text { County }\end{array}$ & 350 & 3.8 & + & - & - & - & - & - & - & - & - & - & 14 & $\begin{array}{l}0.31 \\
\pm \\
0.08\end{array}$ & $\begin{array}{l}205 \\
\pm 95\end{array}$ \\
\hline${ }^{*}$ C. akoensis & .. & .. & 6.0 & - & - & - & - & - & - & + & + & - & + & 12 & $\begin{array}{l}0.41 \\
\pm \\
0.11\end{array}$ & $\begin{array}{l}441 \\
\pm 32\end{array}$ \\
\hline $\begin{array}{l}\text { C. chinensis var. } \\
\text { chinensis }\end{array}$ & $\begin{array}{l}\text { Lising, } \\
\text { Nantou County }\end{array}$ & 1800 & 4.3 & + & - & - & - & - & - & - & - & - & - & 20 & $\begin{array}{l}0.41 \\
\pm \\
0.11\end{array}$ & $\begin{array}{l}104 \\
\pm 36\end{array}$ \\
\hline $\begin{array}{l}\text { C. chinensis var. } \\
\text { chinensis }\end{array}$ & .. & .. & 11.9 & - & + & + & - & $3-5$ & $\begin{array}{l}0.3- \\
0.7\end{array}$ & - & + & - & + & 22 & $\begin{array}{l}0.83 \\
\pm \\
0.19\end{array}$ & $\begin{array}{l}232 \\
\pm 79\end{array}$ \\
\hline $\begin{array}{l}\text { C. chinensis var. } \\
\text { tatushanensis }\end{array}$ & Shalu, Taichung City & 150 & 2.2 & - & - & - & - & - & - & - & - & - & - & 20 & $\begin{array}{l}0.21 \\
\pm \\
0.05\end{array}$ & $\begin{array}{l}25 \pm \\
13\end{array}$ \\
\hline C. crassifolia & $\begin{array}{l}\text { Henglingshan, } \\
\text { Taichung City, }\end{array}$ & 1700 & 4.1 & + & - & - & - & - & - & - & - & - & + & 20 & $\begin{array}{l}0.29 \\
\pm \\
0.06\end{array}$ & $\begin{array}{l}127 \\
\pm 34\end{array}$ \\
\hline C. crassifolia & $\begin{array}{l}\text { Manabangshan,Miaoli } \\
\text { County }\end{array}$ & 1200 & 8.9 & - & - & - & + & $3-6$ & $\begin{array}{l}1.8- \\
3.4\end{array}$ & - & + & - & + & 20 & $\begin{array}{l}0.47 \\
\pm \\
0.11\end{array}$ & $\begin{array}{l}186 \\
\pm 66\end{array}$ \\
\hline${ }^{*} \mathrm{C}$. formosana & $\begin{array}{l}\text { Shoushan, Kaohsiung } \\
\text { City }\end{array}$ & 200 & 3.4 & + & - & - & + & 1 & $\begin{array}{l}0.1- \\
1.3\end{array}$ & - & + & - & + & 12 & $\begin{array}{l}0.41 \\
\pm \\
0.12\end{array}$ & $\begin{array}{l}90 \pm \\
16\end{array}$ \\
\hline${ }^{*} \mathrm{C}$. formosana & .. & .. & 11.3 & - & - & - & + & 2 & $\begin{array}{l}0.3- \\
1.8\end{array}$ & - & + & - & + & 12 & $\begin{array}{l}1.48 \\
\pm \\
1.00\end{array}$ & $\begin{array}{l}138 \\
\pm 46\end{array}$ \\
\hline $\begin{array}{l}\text { *C. } \\
\text { gouriana subsp. } \\
\text { lishanensis }\end{array}$ & $\begin{array}{l}\text { Hehuanshan, Nantou } \\
\text { County }\end{array}$ & 2600 & 6.2 & - & - & - & + & $1-2$ & $\begin{array}{l}0.2- \\
0.7\end{array}$ & - & + & - & + & 12 & $\begin{array}{l}0.81 \\
\pm \\
0.34\end{array}$ & $\begin{array}{l}108 \\
\pm 51\end{array}$ \\
\hline C. grata & Dapu, Chiayi County & 500 & 3.9 & + & - & - & - & - & - & - & - & - & - & 12 & $\begin{array}{l}0.46 \\
\pm \\
0.14\end{array}$ & $\begin{array}{l}89 \pm \\
29\end{array}$ \\
\hline C. grata & .. & .. & 3.5 & - & - & + & - & 1 & $\begin{array}{l}0.1- \\
0.3\end{array}$ & + & - & - & - & 12 & $\begin{array}{l}0.49 \\
\pm \\
0.13\end{array}$ & $\begin{array}{l}108 \\
\pm 22\end{array}$ \\
\hline C. grata & .. & .. & 14.1 & - & + & + & - & 4 & $\begin{array}{l}0.3- \\
1.1\end{array}$ & - & + & - & + & 12 & $\begin{array}{l}1.69 \\
\pm \\
0.69\end{array}$ & $\begin{array}{l}642 \\
\pm \\
208\end{array}$ \\
\hline $\begin{array}{l}\text { C. henryi var. } \\
\text { henryi }\end{array}$ & $\begin{array}{l}\text { Beidawushan, } \\
\text { Pingtung County }\end{array}$ & 1500 & 5.5 & - & - & - & + & $1-3$ & $\begin{array}{l}0.4- \\
0.9\end{array}$ & + & + & - & + & 10 & $\begin{array}{l}0.38 \\
\pm \\
0.07\end{array}$ & $\begin{array}{l}449 \\
\pm \\
131\end{array}$ \\
\hline $\begin{array}{l}{ }^{*} \mathrm{C} \text {. henryi var. } \\
\text { morii }\end{array}$ & $\begin{array}{l}\text { Sinjhongheng, Chiayi } \\
\text { County }\end{array}$ & 2400 & 4.0 & - & - & - & + & 1 & $<0.1$ & + & + & - & + & 10 & $\begin{array}{l}0.58 \\
\pm \\
0.15\end{array}$ & $\begin{array}{l}283 \\
\pm 55\end{array}$ \\
\hline $\begin{array}{l}{ }^{*} \mathrm{C} \text {. henryi var. } \\
\text { morii }\end{array}$ & .. & .. & 7.3 & - & - & - & + & 1 & $\begin{array}{l}0.1- \\
0.2\end{array}$ & + & + & - & + & 11 & $\begin{array}{l}0.90 \\
\pm \\
0.21\end{array}$ & $\begin{array}{l}553 \\
\pm \\
116\end{array}$ \\
\hline C. lasiandra & $\begin{array}{l}\text { Lingmingshan, } \\
\text { Taichung City }\end{array}$ & 3000 & 2.0 & - & - & - & - & - & - & - & - & - & - & 12 & $\begin{array}{l}0.34 \\
\pm \\
0.03\end{array}$ & $\begin{array}{l}96 \pm \\
52\end{array}$ \\
\hline C. lasiandra & $\begin{array}{l}\text { Hehuanshan, Nantou } \\
\text { County }\end{array}$ & 2800 & 19.7 & - & + & + & - & $2-5$ & $\begin{array}{l}0.5- \\
2.7\end{array}$ & - & + & - & - & 14 & $\begin{array}{l}2.76 \\
\pm \\
0.52\end{array}$ & $\begin{array}{l}230 \\
\pm \\
178\end{array}$ \\
\hline $\begin{array}{l}\text { C. } \\
\text { leschenaultiana }\end{array}$ & $\begin{array}{l}\text { Duona trail, } \\
\text { Kaohsiung City }\end{array}$ & 700 & 6.4 & - & - & - & + & 2 & $\begin{array}{l}0.2- \\
0.3\end{array}$ & - & + & - & + & 21 & $\begin{array}{l}0.45 \\
\pm \\
0.14\end{array}$ & $\begin{array}{l}175 \\
\pm 61\end{array}$ \\
\hline
\end{tabular}

Noted: *: endemic species; LO: locality; EL: elevation; SD: stem diameter; PF: phloem fibers in dense bundles or strands; AR: arc-like bark; COG: cogwheel-like r rhytidome; RHL: rhytidome layer number; RHT: rhytidome thickness; WER: wedge-like rays; ID: indentation of the axial parenchyma near the wider rays; VR: ve dilatations; VB: number of vascular bundle; $X W$ : xylem width (mean $\pm S D, n=x y l e m$ number); RW: ray width (mean $\pm S D, n=$ ray number); WR: wide rays initiat initiated abruptly; RP: ring-porous vessel; SRP: semi-ring-porous vessel; DS: developmental stage (1: first stage, collenchyma or primary phloem fibres present beneath the primary phloem, secondary phloem fibers present. 3: third stage, sequent periderm develop within the secondary phloem, wider rays and larger v $\epsilon$ sequent periderm developed in deeper layers in the secondary phloem, narrow rays present). 


\begin{tabular}{|lllllllllllllllll}
\hline Characters & LO & EL & SD & PF & AR & COG & SE & RHL & RHT & WER & ID & VR & RD & VB & XW & RW \\
Scientific name & & $(\mathrm{m})$ & $(\mathrm{mm})$ & & & & & $($ no. $)$ & $(\mathrm{mm})$ & & & & & $(\mathrm{no})$. & $(\mathrm{mm})$ & $(\mu \mathrm{m})$
\end{tabular}

\begin{tabular}{|c|c|c|c|c|c|c|c|c|c|c|c|c|c|c|c|c|}
\hline $\begin{array}{l}\text { C. } \\
\text { leschenaultiana }\end{array}$ & .. & .. & 7.8 & - & - & - & + & 2 & $\begin{array}{l}0.2- \\
0.5\end{array}$ & - & + & - & + & 16 & $\begin{array}{l}0.69 \\
\pm \\
0.32\end{array}$ & $\begin{array}{l}145 \\
\pm 52\end{array}$ \\
\hline $\begin{array}{l}\text { C. } \\
\text { leschenaultiana }\end{array}$ & .. & .. & 15.6 & - & - & - & + & 4 & $\begin{array}{l}0.2- \\
1.6\end{array}$ & - & + & - & + & 19 & $\begin{array}{l}1.34 \\
\pm \\
0.64\end{array}$ & $\begin{array}{l}225 \\
\pm 46\end{array}$ \\
\hline C. meyeniana & $\begin{array}{l}\text { Duona trail, } \\
\text { Kaohsiung City }\end{array}$ & 700 & 7.3 & - & - & - & + & 3 & $\begin{array}{l}0.4- \\
0.8\end{array}$ & - & + & - & + & 21 & $\begin{array}{l}0.58 \\
\pm \\
0.12\end{array}$ & $\begin{array}{l}117 \\
\pm 39\end{array}$ \\
\hline C. montana & $\begin{array}{l}\text { Hehuanshan, Nantou } \\
\text { County }\end{array}$ & 3200 & 3.1 & + & - & - & + & 1 & $\begin{array}{l}0.2- \\
0.3\end{array}$ & - & - & - & + & 12 & $\begin{array}{l}0.34 \\
\pm \\
0.06\end{array}$ & $\begin{array}{l}202 \\
\pm 29\end{array}$ \\
\hline $\begin{array}{l}{ }^{*} \text { C. } \\
\text { parviloba subsp. } \\
\text { bartlettii }\end{array}$ & $\begin{array}{l}\text { Hehuan river, Nantou } \\
\text { County }\end{array}$ & 2600 & 2.4 & - & - & - & + & 1 & $\begin{array}{l}0.1- \\
0.2\end{array}$ & - & - & - & + & 12 & $\begin{array}{l}0.32 \\
\pm \\
0.10\end{array}$ & $\begin{array}{l}64 \pm \\
25\end{array}$ \\
\hline
\end{tabular}

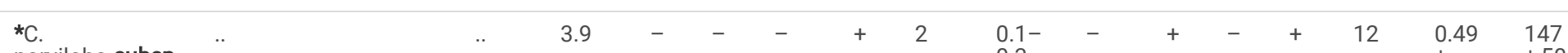

$\begin{array}{lll}\text { parviloba subsp. } & 0.3 & \pm \\ & \pm 50 & 0.13 \quad\end{array}$

bartlettii

\begin{tabular}{|c|c|c|c|c|c|c|c|c|c|c|c|c|c|c|c|c|}
\hline $\begin{array}{l}\text { C. } \\
\text { pseudootophora }\end{array}$ & $\begin{array}{l}\text { Sihyuanyakou, Yilan } \\
\text { County }\end{array}$ & 1800 & 3.5 & - & + & + & - & 1 & $\begin{array}{l}0.1- \\
0.2\end{array}$ & + & + & - & - & 6 & $\begin{array}{l}0.93 \\
\pm \\
0.10\end{array}$ & $\begin{array}{l}393 \\
\pm 87\end{array}$ \\
\hline${ }^{*}$ C. psilandra & Alishan, Chiayi County & 2300 & 4.3 & - & - & - & - & - & - & - & - & + & - & 20 & $\begin{array}{l}0.33 \\
\pm \\
0.11\end{array}$ & $\begin{array}{l}387 \\
\pm 99\end{array}$ \\
\hline${ }^{*}$ C. psilandra & & .. & 4.9 & - & - & - & - & - & - & - & - & + & - & 23 & $\begin{array}{l}0.20 \\
\pm \\
0.08\end{array}$ & $\begin{array}{l}218 \\
\pm 79\end{array}$ \\
\hline${ }^{*}$ C. tamurae & $\begin{array}{l}\text { Fonggangshan, } \\
\text { Kaohsiung City }\end{array}$ & 1600 & 2.1 & + & - & - & - & - & - & - & - & - & - & 12 & $\begin{array}{l}0.22 \\
\pm \\
0.05\end{array}$ & $\begin{array}{l}107 \\
\pm 23\end{array}$ \\
\hline${ }^{*}$ C. tamurae & .. & .. & 4.5 & - & + & + & - & 1 & $\begin{array}{l}0.1- \\
0.3\end{array}$ & - & + & - & + & 12 & $\begin{array}{l}0.52 \\
\pm \\
0.14\end{array}$ & $\begin{array}{l}167 \\
\pm 49\end{array}$ \\
\hline${ }^{*} \mathrm{C}$. tamurae & & .. & 8.3 & - & + & + & - & $3-4$ & $\begin{array}{l}0.1- \\
1.2\end{array}$ & - & + & - & + & 12 & $\begin{array}{l}0.91 \\
\pm \\
0.13\end{array}$ & $\begin{array}{l}393 \\
\pm 61\end{array}$ \\
\hline${ }^{*}$ C. tashiroi & $\begin{array}{l}\text { Rueisuei trail, Hualien } \\
\text { County }\end{array}$ & 2400 & 4.0 & + & - & - & - & - & - & - & - & - & - & 14 & $\begin{array}{l}0.32 \\
\pm \\
0.07\end{array}$ & $\begin{array}{l}289 \\
\pm \\
116\end{array}$ \\
\hline${ }^{{ }^{*} \text { C. tashiroi }}$ & $\begin{array}{l}\text { Beizihtong forest trail, } \\
\text { Chiayi county }\end{array}$ & 1600 & 8.8 & - & - & - & - & - & - & - & + & - & + & 20 & $\begin{array}{l}0.58 \\
\pm \\
0.10\end{array}$ & $\begin{array}{l}343 \\
\pm \\
155\end{array}$ \\
\hline${ }^{*}$ C. tashiroi &.. & .. & 17.8 & - & - & - & - & - & - & - & + & - & + & 20 & $\begin{array}{l}1.44 \\
\pm \\
0.30\end{array}$ & $\begin{array}{l}450 \\
\pm \\
187\end{array}$ \\
\hline $\begin{array}{l}{ }^{*} \text { C. terniflora var. } \\
\text { garanbiensis }\end{array}$ & $\begin{array}{l}\text { Hengchun, Pingtung } \\
\text { County }\end{array}$ & 100 & 2.7 & + & - & - & - & - & - & - & - & - & - & 14 & $\begin{array}{l}0.33 \\
\pm \\
0.09\end{array}$ & $\begin{array}{l}63 \pm \\
29\end{array}$ \\
\hline
\end{tabular}

Noted: *: endemic species; LO: locality; EL: elevation; SD: stem diameter; PF: phloem fibers in dense bundles or strands; AR: arc-like bark; COG: cogwheel-like r rhytidome; RHL: rhytidome layer number; RHT: rhytidome thickness; WER: wedge-like rays; ID: indentation of the axial parenchyma near the wider rays; VR: ve dilatations; VB: number of vascular bundle; $X W$ : xylem width (mean $\pm S D, n=x y l e m$ number); RW: ray width (mean $\pm S D, n=$ ray number); WR: wide rays initiat initiated abruptly; RP: ring-porous vessel; SRP: semi-ring-porous vessel; DS: developmental stage (1: first stage, collenchyma or primary phloem fibres present beneath the primary phloem, secondary phloem fibers present. 3: third stage, sequent periderm develop within the secondary phloem, wider rays and larger v $\epsilon$ sequent periderm developed in deeper layers in the secondary phloem, narrow rays present). 


\begin{tabular}{|c|c|c|c|c|c|c|c|c|c|c|c|c|c|c|c|c|}
\hline $\begin{array}{l}\text { Characters } \\
\text { Scientific name }\end{array}$ & LO & $\begin{array}{l}\text { EL } \\
(m)\end{array}$ & $\begin{array}{l}\text { SD } \\
(\mathrm{mm})\end{array}$ & PF & AR & COG & SE & $\begin{array}{l}\text { RHL } \\
\text { (no.) }\end{array}$ & $\begin{array}{l}\text { RHT } \\
(\mathrm{mm})\end{array}$ & WER & ID & VR & RD & $\begin{array}{l}\text { VB } \\
\text { (no.) }\end{array}$ & $\begin{array}{l}X W \\
(\mathrm{~mm})\end{array}$ & $\begin{array}{l}\text { RW } \\
(\mu \mathrm{m})\end{array}$ \\
\hline $\begin{array}{l}\text { *C. terniflora var. } \\
\text { garanbiensis }\end{array}$ & .. & .. & 2.8 & + & + & + & - & 1 & $\begin{array}{l}0.1- \\
0.2\end{array}$ & - & + & - & + & 12 & $\begin{array}{l}0.34 \\
\pm \\
0.05\end{array}$ & $\begin{array}{l}77 \pm \\
31\end{array}$ \\
\hline $\begin{array}{l}\text { *C. terniflora var. } \\
\text { garanbiensis }\end{array}$ & .. & .. & 4.3 & - & + & + & - & $1-2$ & $\begin{array}{l}0.2- \\
0.5\end{array}$ & - & + & - & + & 19 & $\begin{array}{l}0.36 \\
\pm \\
0.10\end{array}$ & $\begin{array}{l}77 \pm \\
34\end{array}$ \\
\hline${ }^{*} \mathrm{C}$. tsugetorum & $\begin{array}{l}\text { Hehuanshan, Nantou } \\
\text { County }\end{array}$ & 3200 & 3.9 & - & - & - & - & - & - & - & - & + & - & 17 & $\begin{array}{l}0.17 \\
\pm \\
0.08\end{array}$ & $\begin{array}{l}374 \\
\pm \\
131\end{array}$ \\
\hline $\begin{array}{l}\text { C. uncinata var. } \\
\text { okinawensis }\end{array}$ & $\begin{array}{l}\text { Dahanshan, Pingtung } \\
\text { County }\end{array}$ & 900 & 4.0 & + & - & - & - & - & - & - & - & - & - & 14 & $\begin{array}{l}0.31 \\
\pm \\
0.08\end{array}$ & $\begin{array}{l}195 \\
\pm 84\end{array}$ \\
\hline $\begin{array}{l}\text { C. uncinata var. } \\
\text { okinawensis }\end{array}$ & .. & .. & 9.7 & - & + & + & - & $1-3$ & $\begin{array}{l}0.5- \\
1.9\end{array}$ & - & + & - & + & 12 & $\begin{array}{l}0.12 \\
\pm \\
0.20\end{array}$ & $\begin{array}{l}332 \\
\pm 83\end{array}$ \\
\hline $\begin{array}{l}\text { C. uncinata var. } \\
\text { uncinata }\end{array}$ & $\begin{array}{l}\text { Syuejian, Miaoli } \\
\text { County }\end{array}$ & 2000 & 5.7 & + & - & - & - & - & - & - & - & - & + & 12 & $\begin{array}{l}0.73 \\
\pm \\
0.07\end{array}$ & $\begin{array}{l}260 \\
\pm 61\end{array}$ \\
\hline $\begin{array}{l}\text { C. uncinata var. } \\
\text { uncinata }\end{array}$ & $\begin{array}{l}\text { Shihkejianshan, } \\
\text { Pingtung County }\end{array}$ & 1300 & 12.4 & - & + & + & - & $3-5$ & $\begin{array}{l}0.5- \\
1.5\end{array}$ & - & + & - & + & 14 & $\begin{array}{l}1.38 \\
\pm \\
0.19\end{array}$ & $\begin{array}{l}386 \\
\pm 62\end{array}$ \\
\hline Sum & & & & 12 & 8 & 8 & 9 & & & 5 & 18 & 2 & 15 & & & \\
\hline
\end{tabular}

Noted: *: endemic species; LO: locality; EL: elevation; SD: stem diameter; PF: phloem fibers in dense bundles or strands; AR: arc-like bark; COG: cogwheel-like r rhytidome; RHL: rhytidome layer number; RHT: rhytidome thickness; WER: wedge-like rays; ID: indentation of the axial parenchyma near the wider rays; VR: ve dilatations; VB: number of vascular bundle; XW: xylem width (mean $\pm S D, n=x y l e m$ number); RW: ray width (mean $\pm S D, n=$ ray number); WR: wide rays initiat initiated abruptly; RP: ring-porous vessel; SRP: semi-ring-porous vessel; DS: developmental stage (1: first stage, collenchyma or primary phloem fibres present beneath the primary phloem, secondary phloem fibers present. 3: third stage, sequent periderm develop within the secondary phloem, wider rays and larger v $\epsilon$ sequent periderm developed in deeper layers in the secondary phloem, narrow rays present).

The ontogenetic stage of all 22 Clematis species was determined based on two lianas and one herb reported in Isnard et al. (2003a, b). During the first ontogenetic stage, stems have a cortex and a vascular cylinder, collenchyma and primary sclerenchyma fibers (primary phloem fibers) as a continuous ring or dense bundles in liana, but as separate bundle sheaths in herb. The primary phloem fibers are located between the collenchyma and secondary phloem. During the second stage, the stem has secondary tissue and an initial periderm (Esau 1958) formed beneath the primary phloem in liana, but cortex suberisation and parenchyma lignified in herb. Secondary phloem fibers are produced along the secondary phloem. In the third stage, sequent periderms develop within the secondary phloem; the cortex and primary phloem are detached into a ringbark or a continuous segment (Esau 1958); possess a wide ray and forms many larger vessels; secondary phloem fibers increases. In the last stage, sequent periderm is produced in progressively deeper layers in the secondary phloem; periderms are detached after forming decorticated tissues.

\section{Results}

\section{Bark Morphologies}

The anatomical and morphological details of stems for the 22 species investigated in this study are listed in Tables 2 and presented in Figs. 1 to 7 . Seventeen species had wrinkled rhytidome; among them, nine had continuous segment rhytidome, and eight had cogwheel-like rhytidome, which peeled and teared easily. The rhytidome of $C$. crassifolia (Fig. 2A) was a continuous segment and was the thickest (1.8-3.4 mm), that of $C$. lasiandra (Fig. 3F) was cogwheel-like with 0.5-2.7 mm thickness (Table 2). The remaining five species, C. akoensis (Fig. 1B), C. chinensis var. tatushanensis (Fig. 1E), C. psilandra (Fig. 5C-D), C. tashiroi (Fig. 6B-D), and C. tsugetorum (Fig. 7B), were without rhytidome.

\section{Cambial Variant Types}

The stems of the 22 species investigated were shallowly grooved or angulated, and the stems were round (Fig. 1A-B) or hexagon and deeply grooved in shape (Figs. 2E, 3E, 5B, 5E) in the younger stem. Only C. henryi var. morii (Fig. 3D) was a lobe with asymmetrical conformation. The stem of $C$. formosana (Fig. 2C) was eccentric to oval or elliptical. C. gouriana subsp. lishanensis (Figs. 2D) formed discontinuous wedge-like phloem. The interfascicular cambia made the rays wider and wedge-like (Schweingruber et al. 2011); this character was apparent in five of the Clematis species studied, such as $C$. akoensis (Fig. 1B), $C$. grata (Fig. 3A), C. henryi var. henryi (Fig. 3B), C. henryi var. morii (Fig. 3D), and C. pseudootophora (Fig. 5B) (Table 2, WER column). For example, C. henryi var. henryi and $C$. grata had the widest rays, with a maximum width of $668 \mu \mathrm{m}$ and $642 \mu \mathrm{m}$, respectively (Table 2, RW column). The rays size of five Clematis species, C. chinensis var. chinensis (Fig. 1D), C. crassifolia (Fig. 2A), C. grata, C. leschenaultiana (Fig. 4C), and C. tashiroi (Fig. 6D), became wider which called as abrupt wide rays which defined in Carlquist (1995). The remaining 13 Clematis species examined generally developed axial vascular elements in segments, and separated by the wider xylem and phloem rays, forming xylem in plate variant.

\section{Variation in Vascular Elements}


There was an obvious indentation in the region of the wider rays, except in C. chinensis var. tatushanensis (Fig. 1E), C. montana (Fig. 4E), C. psilandra (Fig. 5D), and C. tsugetorum (Fig. 7B). Perhaps, this is because the stem diameters of $C$. chinensis var. tatushanensis, $C$. montana were too small to develop wider rays. The wedge-shaped indentations developed in the interfascicular region were only found in $C$. henryi var. morii due to its thin-walled axial parenchyma adjacent to the thin-walled ray cells (Sieber and Kucera 1980). The axial parenchyma of $C$. psilandra and $C$. tsugetorum were very scarce, and vessel distribution was limited to the central portions of the fascicular area with growth rings. Almost all Clematis species in this study had semi-ring-porous vessels, except for $C$. crassifolia (Fig. 2A) and C. lasiandra (Fig. 3F), which had ring-porous vessels with distinct annual rings. None of the species had diffuseporous vessels, which is consistent with previous reports (Schweingruber et al. 2011).

An arc-like bark developed in eight species, C. chinensis var. chinensis (Fig. 1D), C. grata (Fig. 2F, 3A), C. lasiandra (Fig. 3F), C. pseudootophora (Fig. 5B), C. tamurae (Fig. 6A), C. terniflora var. garanbiensis (Figs. 6E-F, 7A), C. uncinata var. okinawensis (Fig. 7C-D), and C. uncinata var. uncinata (Fig. 7E-F) (Table 2, AR column). C. uncinata var. uncinata (Fig. 7E-F) had a primary xylem ring located around the pith. The pith cavity formed in $C$. parviloba subsp. bartlettii (Fig. 4F, 5A), C. psilandra (Fig. 5C), C. uncinata var. okinawensis (Fig. 7C), and C. uncinata var. uncinata (Fig. 7F), among them, $C$. uncinata var. uncinata formed a hexagon shape.

Stem cross-sections showed that nine Clematis species formed one to three narrow rays within some secondary xylem tissues [C. chinensis var. chinensis (Fig. 1D), C. formosana (Fig. 2B-C), C. gouriana subsp. lishanensis (Figs. 2D), C. grata (Fig. 3A), C. lasiandra (Fig. 3F), C. leschenaultiana (Fig. 4C), C. tashiroi (Fig. 6D), C. uncinata var. okinawensis (Fig. 7C-D), and C. uncinata var. uncinata (Fig. 7E-F)] (Table 2, NR column).

In this study, the number of vascular bundles observed in Clematis species ranged from 6-21. Of which, three were classified as few type (14\%), 13 as many type (59\%), and six as central type (27\%) (Table 2, VB column). Only one species, C. pseudootophora (Fig. 5B), had six vascular bundles.

\section{Developmental Stages}

Based on the characteristics observed in stem cross-sections of 42 samples from the 22 species, stem developments were divided into four ontogenetic stages (Table 2, DS column). If multiple samples of the same species were collected (which was the case for 15 species), each sample was investigated separately. Therefore, these species, such as $C$. grata (Figs. 2E-F, 3A) and C. leschenaultiana (Figs. 4A-C), were assigned one to four developmental stages. The remaining seven species with only one sample were assigned with one developmental stage. No rhytidomes were found in six samples consisting of three species, C. psilandra (Figs. 5C-D), C. tashiroi (Figs. 6B-D), and C. tsugetorum (Fig. 7B).

Nine samples consisting of nine species had dense or strand bundles of primary phloem fibers within the cortex and were identified as being in the first developmental stage (Figs. 1A, 1C, 1E, 1F, 2E, 5E, 6E, 7C, 7E). In the second stage, seven samples consisting of seven species had the ring-like initial periderm and secondary phloem fibers (Figs. 1B, 2B, 2F, 3E, 4E-F, 6F). In the third stage, seven samples consisting of seven species had loss of primary fibers; sequent periderms were produced within the secondary phloem; cork and non-conducting phloem were detached into a rhytidome; wide rays, larger vessels (Figs. $3 \mathrm{~B}-\mathrm{C}$, $4 A, 4 D, 5 A, 5 B, 5 F)$. In the fourth stage, thirteen samples consisting of twelve species had sequent periderms produced continuously with progressively deeper layers, and narrow rays were formed (Figs. 1D, 2A, 2C-D, 3A, 3D, 3F, 4B-C, 6A, 7A, 7D, 7F).

Rhytidomes were not observed in three samples of $C$. tashiroi, and its epidermis was deep green in color and glabrous; the stem cross-section was hexagon in shape. However, according to the stem features, three developmental stages of $C$. tashiroi can be divided. In the first stage, the stem had primary phloem fibers as strands along the secondary phloem (Figs. 6B). In the second stage, secondary phloem became triangular with secondary phloem fibers, and a wider ray and many larger vessels were formed (Figs. 6C). In the third stage, rays became unequal in width, and narrow rays were formed (Figs. 6D).

\section{Bracket key to 22 clematis species}

A key to the 22 Clematis species in Taiwan, based on the characteristics of bark and vascular bundles, is provided below.

1. Vessels restricted to the central portions of the fascicular area.........................2

1. Vessels unrestricted to the central portions of the fascicular area........................

2. Vascular bundles (20), pith cavity................................ Clematis psilandra

2. Vascular bundles (17), pith without cavity... ... Clematis tsugetorum

3. Wedge-like phloem Clematis gouriana subsp. lishanensis

3. Non-wedge-like phloem ..............................................................4

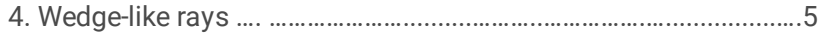

4. Non-wedge-like rays................................................................

5. Vascular bundles (6)......................................elematis pseudootophora

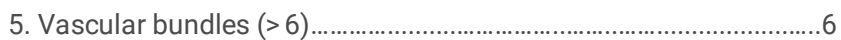

6. Arc-like bark ............................................................elematis grata

6. Non-arc-like bark .... ....................................................................... 
8. Bark without rhytidome layers.

.Clematis akoensis

8. Bark with one to three rhytidome layers

Clematis henryi var. henryi

9. Bark without rhytidome layers. . .10

9. Bark with one to five rhytidome layers .11

10. Vascular bundles (20). Clematis chinensis var. tatushanensis

10. Vascular bundles (14-20) ... Clematis tashiroi

11. Pith cavity. Clematis parviloba subsp. bartlettii

11. Pith without cavity .12

12. Non-arc-like bark. . .13

12. Bark arc-like. 17

13. Vascular bundles (12) .14

13. Vascular bundles (16-21) .15

14. Stem, eccentric to oval or elliptical. Clematis formosana

14. Stem, round. Clematis montana

15. Rhytidome thickness $(0.4-0.8 \mathrm{~mm})$. ..Clematis meyeniana

15. Rhytidome thickness $(>0.9 \mathrm{~mm})$. .16

16. Rhytidome thickness $(0.2-1.6 \mathrm{~mm})$. Clematis leschenaultiana

16. Rhytidome thickness (1.8-3.4 mm). Clematis crassifolia

17. Vessels, ring-porous. Clematis lasiandra

17. Vessels, semi-ring-porous. .18

18. Pith cavity, hexagon in shape Clematis uncinata var. uncinata

18. Pith without cavity. . .19

19. Vascular bundles (20-22) Clematis chinensis var. chinensis

19. Vascular bundles (12-19) 20

20. Constant vascular bundles (12) Clematis tamurae

20. Varying vascular bundles. .21

21. Narrow rays not formed. Clematis terniflora var. garanbiensis

21. Narrow rays approximately 13. Clematis uncinata var. okinawensis

\section{Discussion}

The rhytidome is one of the diagnostic characteristics of the Clematis genus. The texture and detachment of the rhytidome result from an irregular appearance in the developmental stages. It is easier to correctly identify the structure of rhytidome from fresh materials than from dry materials. Among the 22 species, the rhytidome was not observed in C. akoensis, C. chinensis var. tatushanensis, C. psilandra, C. tashiroi, and C. tsugetorum (Table 2 ) due to their smaller stem diameters or earlier production of periderm establishing inner to the cortex. The stem diameters of some Clematis species were smaller than 8.8 $\mathrm{mm}$ and can form a rhytidome, but that of $C$. tashiroi was approximately $17.8 \mathrm{~mm}$ and did not appear to have a rhytidome. We simply suggested that rhytidome was not observed even in some species with smaller stems already having this feature. Perhaps there is an evolutionary mechanism (e.g., heterochrony) leading to the delayed formation of rhytidome in $C$. tashiroi. However, their bark may continue to develop into one of the rhytidome types; therefore, continued observations are necessary. 
The stem cross-section of $C$. henryi var. morii and $C$. formosana were a lobe with asymmetrical conformation and eccentric to oval or elliptical, respectively. This generates as an irregular conformation, found in some Apocynaceae and Malpighiaceae (Angyalossy et al. 2015). Clematis gouriana subsp. lishanensis forms discontinuous wedge-like phloem, a common characteristic of the family Bignoniaceae (Pace et al. 2011). In summary, except for xylem in plate variant, two cambial variants are found in the Ranunculaceae family-stems with an irregular conformation, and phloem arcs/wedges.

Indentation toward the pith in the wider rays region is obvious in Clematis species (Sieber and Kucera 1980; Carlquist 1995). This feature was found in most Clematis species investigated in this study, except for those with a small stem diameter (Table 2, ID column). The bark texture formed an arc shape outside the fascicular region in C. vitalba (Sieber and Kucera 1980); this feature was also found in eight species apparently (Table 2, AR column). The arc feature comprised several layers of periderm combined with the non-conducting phloem (e.g., Fig. 1D. The characteristic of arc-like bark indeed is diagnostic evidence for Clematis species comparison.

The vessel restriction pattern was found in Xanthorhiza apiifolia of Ranunculaceae (Carlquist 1995), Valeriana of Valerianaceae (Carlquist 1983), Launea of Astraceae (Carlquist 1988), and Dicentra and Hunnemannia of Papaveraceae. This pattern appeared in Clematis psilandra (Fig. 5C-D) and C. tsugetorum (Fig. 7B) studied, but few vessels were in contact with the rays; this phenomenon also appeared in the Clematis genus (Carlquist and Zona 1988). Carlquist and Zona (1988) suggested that the species clearly showed vessel restriction patterns; stems had limited diameter and duration, and they were either woody herbs or short-lived shrubs. This unusual wood feature of Clematis psilandra and $C$. tsugetorum was useful as a potential indicator of relationship (Carlquist 1995).

Clematis species often have 12 vascular bundles, with Smith (1928) finding this feature in $67 \%$ of 138 species studied. This is generally considered the central type for this genus. In Taiwan, only $27 \%$ of the 22 Clematis species had 12 vascular bundles. Five of the species in our study had inconsistent numbers of vascular bundles compared with the findings of Smith (1928). In our study, Clematis henryi var. henryi (Fig. 3B) had 10 vascular bundles, but only six were recorded by Smith (1928). Moreover, C. chinensis var. chinensis (Fig. 1C-D) had $20-23$ bundles, C. lasiandra (Fig. 3F) had 12-14, C. meyeniana (Fig. 4D) had 21, and both C. uncinata var. okinawensis (Fig. 7C, D) and C. uncinata var. uncinata (Fig. 7E, F) had 12-14, but Smith (1928) recorded only 12 vascular bundles in these species. The inconsistent results might be due to different sample sizes, or developmental stages; further investigation is required to elucidate this discrepancy.

Vascular bundle numbers are usually constant for a given species, but the numbers can vary in some species. We examined three samples of $C$. leschenaultiana (Fig. 4A-C) collected from the same branch, finding that the number of vascular bundles varied in different growth stages. The vascular bundle number of $C$. leschenaultiana is from 21 (Figs. 4A) to 16 (Figs. 4B) to 19 (Figs. 4C). We checked that within 21 vascular bundles in the second stage, 10 might aggregate closely into five groups, each group was composed of a big and a small vascular bundle, and the vascular bundle seems to be 16 . In the fourth stage, some groups gradually separated, the number of vascular bundles seems to change to be 19 . These processes could indicate that the bigger vascular bundle and fascicular zone were more active than the smaller vascular bundle and interfascicular zone. To confirm this, future work should focus on collecting and observing more specimens from different stem positions of the same species.

Clematis henryi is taxonomically treated as $C$. henryi var. henryi and $C$. henryi var. morii (Yang and Huang 1996), but $C$. henryi var. morii is sometimes treated as a species, $C$. morii (Wang and Bartholomew 2007). In this study, these two taxa had wedge-like rays and a continuous rhytidome segment, but the stem of $C$. henryi var. henryi was round while that of $C$. henryi var. morii was an irregular conformation variant. These stem shape characteristics provide a way to distinguish these two taxa.

The site of phellogen initiation is often in an outer layer of cortical parenchyma cells, one or two layers beneath the epidermis (Beck, 2011). However, the phellogen location was highly variable and formed deep within the cortex, in the outer secondary phloem, or even within the secondary phloem (Isnard et al. 2003a), such as the Clematis genus. Compared with the characteristics of the developmental stage described in Isnard et al. (2003a, b), some features were different or could not be found in the present study. In the first stage, the stem had the primary phloem fibers in dense bundles or strands, not in a continuous ring; and in the fourth stage, narrow rays were found.

The one to two samples of two shrub species, C. psilandra, and C. tsugetorum, were not enough to assess their developmental stages. Assessing their development stage by the features of lianas and herb (Isnard et al. 2003a, b), we suggested they should belong to the fourth stage due to develop the narrow rays. However, further study collecting specimens of these species and examining their characteristics of different developmental stages is needed, especially species without the rhytidome.

In the present study, different narrow rays were observed in nine Clematis species (Table 2, NR column). These rays had been recorded in the species Aristolochia macrophylla (Aristolochiaceae) (Carlquist 1993; Schweingruber et al. 2011), in the Cucurbitaceae family (Carlquist 1992), and the Cyclea genus (Menispermaceae) (Yang and Chen 2016). The characteristics of narrow rays and activity of the bigger vascular bundles and the fascicular areas of $C$. grata are similar to those of $C$. vitalba, showing that they could be related to stem bark thickness and the formation of arc-like or cogwheel-like bark. However, the narrow rays were not found in all of the species investigated in our study, and its presence might be related to stem diameter size, but this needs further investigation.

The pith cavity resulted from non-lignified walls in the inner-most pith parenchyma cells; this characteristic was found in four species, Clematis parviloba subsp. bartlettii, C. psilandra, C. uncinata var. okinawensis, and C. uncinata var. uncinata.

Moreover, the stem features of the Clematis genus, such as the arc-like bark, rhytidome, wedge-like phloem, ray, and indentations, restricted vessel pattern, secondary phloem fibers, and narrow rays, are very important diagnostic characteristics used to identify the species in the Clematis genus. The stem anatomy of the 22 Clematis species described here provided evidence for systematic problems within this genus.

Page $10 / 19$ 


\section{Conclusions}

The objectives of this study were to understand the stem anatomy of the 22 Taiwanese Clematis species in the Ranunculaceae family. Our results showed that due to successive layers of periderm combined with non-conducting phloem, the cogwheel-like and continuous segments rhytidome were formed in most Clematis species. Three cambial variants of the Clematis genus were found-stems with an irregular conformation, wedge-like phloem, and xylem in plate. Most Clematis species had the indentation of the axial parenchyma near the wider rays, ray dilatation, and varied in the numbers and sizes of vascular bundles. The vessel restriction pattern was found in two shrubs, $C$. psilandra and $C$. tsugetorum, which were different from the other vine Clematis species. The narrow rays occurred in some secondary xylem, and no diffuse-porous vessels dispersed throughout the stems. Further fresh materials collection and observations of different developmental stages are still needed. In conclusion, the data presented here provide important basic information on lianas addressing Clematis taxonomy to understand their morphology and ensure the conservation of their diversity.

\section{Declarations}

Ethics approval and consent to participate

Not applicable

Consent for publication

Not applicable

Availability of data and material

Not applicable

Competing interests

The authors declare that they have no competing interests

Authors' contributions

SZY conceived of and designed the experiments and wrote the paper. PHC conducted the fieldwork and collected the plant specimens and performed the taxonomical study. CFC analyzed and interpreted the plants growth stage regarding the patterns of secondary growth of stem cross-section. Three authors read and approved the final manuscript

Acknowledgements

We thank the herbaria TAI and TAIF for examining the specimens, and Dr. Tsung-Yu Aleck Yang for suggestions improved this manuscript. We truly are thankful to the staff members of the herbarium PPI at the National Pingtung University of Science and Technology for assistance with lianas specimen collection and photography.

\section{References}

Angyalossy V, Angeles G, Pace MR, Lima AC, Dias-Leme CL, Lohmann LG, Madero-Vega C (2012) An overview of the anatomy, development and evolution of the vascular system of lianas. Plant Ecol Divers 5: 167-182.

Angyalossy V, Pace MR, Lima AC (2015) Liana Anatomy: a Broad Perspective on Structural Evolution of the Vascular System. In: Schnitzer SA, Bongers F, Burnham RJ, Putz FE (eds.) Ecology of Lianas. Wiley Blackwell, West Sussex, U.K. pp. 253-287.

Beck CB (2011) An Introduction to Plant Structure and Development: Plant Anatomy for the Twenty-First Century. 2nd Edition, Cambridge University Press, Cambridge.

Carlquist S (1983) Wood anatomy of Calyceraceae and Valerianaceae, with comments on aberrant perforation plates in predominantly herbaceous groups of dicotyledons. Aliso 10: 413-425.

Carlquist S (1988) Comparative Wood Anatomy. Springer Verlag, Berlin \& Heidelberg. 436 pp.

Carlquist S (1991) Anatomy of Vine and Liana Stems: a Review and Synthesis. In: Putz FE, Mooney HA (eds.) The Biology of Vines. Cambridge University Press. UK. pp 53-71

Carlquist S (1992) Woody anatomy of selected Cucurbitaceae and its relationship to habit and systematics. Nordic J Bot 12:347-355

Carlquist S (1993) Wood and bark anatomy of Aristolochiaceae: systematic and habitual correlations. IAWA J 14:341-357

Carlquist S (1995) Wood and bark anatomy of Ranunculaceae (including Hydrastis) and Glaucidiaceae. Aliso 14:65-84.

Carlquist S (2001) Cambial Variants (Anomalous Secondary Growth). In: Carlquist S (ed.) Comparative Wood Anatomy, 2nd edn. Systematic, Ecological and Evolutionary Aspects of Dicotyledon Wood. Spinger-Verlag Press, Berlin, Germany. pp 271-295

Page $11 / 19$ 
Esau K (1958) Plants Anatomy. New York, John Wiley \& Sons, Inc.

Evert RF (2006) Esau's Plant Anatomy. Meristems, Cells and Tissues of the Plant Body. Their Structure, Function and Development. Wiley and Sons. Hoboken, $\mathrm{NJ}$, USA.

Ferreira T, Rasband W (2011) The ImageJ User Guide Version 1.44. http://rsbweb.nih.gov/ij/docs/user-guide.pdf

Gregory M (1994) Bibliography of Systematic Wood Anatomy of Dicotyledons. IAWA J Suppl 1.

lawa Committee on Nomenclature (1964) Multilingual Glossary of Terms Used in Wood Anatomy. Verlagsbuchanstalt Konkordia, Winterthur, Switzerland. $\mathrm{p}$ 186

Isnard S, Rowe N, Speck T (2003a) Growth habit and mechanical architecture of the sand dune-adapted climber Clematis flammula L. var. maritima L. Ann Bot 91(4):407-417

Isnard S, Speck T, Rowe NP (2003b) Mechanical architecture and development in Clematis: implications for canalised evolution of growth forms. New Phytol 158:543-559

Mauseth JD (1988) Plant Anatomy. The Benjamin/Cummings Publishing Company, Menlo Park, CA.

Pace MR, Lohmann LG, Angyalossy V (2011) Evolution of disparity between the regular and variant phloem in Bignonieae (Bignoniaceae). Am J Bot 98:602618

Rajput KS, Chaudhary BD, Patil VS (2014) Development of successive cambia and structure of secondary xylem of Ipomoea obscura (Convolvulaceae). Pol Bot J 59:55-61 https://doi.org/10.2478/pbj-2014-0009

Schweingruber FH, Börner A, Schulze ED (2011) Ranunculaceae. In: Schweingruber FH et al. (eds.) Atlas of Stem Anatomy in Herbs, Shrubs and Trees. vol. 1. Springer-Verlag, Berlin Heidelberg. pp 352-371

Sieber M, Kucera LJ (1980) On the stem anatomy of Clematis vitalba L. IAWA Bulletin, vol. I (1-2):49-54

Smith P (1928) A comparative study of the stem structure of the genus Clematis with special reference to anatomical changes induced by vegetative propagation. Trans R Soc Edinb Earth Sci 70:643-664

Yang SZ, Chen PH (2015) Classifying Taiwan lianas with radiating plates of xylem. Taiwania 60:151-159

Yang SZ, Chen PH (2016) Cambial variants in the Family Menispermaceae in Taiwan. Am J Plant Sci 7:841-854

Yang TYA, Huang TC (1996) Convolvulaceae. In: Huang TC, et al. (eds.) Flora of Taiwan, 2nd edn. Editorial Committee, Dept Bot, NTU, Taipei, pp 515-545

Wang W, Bartholomew B (2007) Clematis. In: Wu ZY, Raven PH \& Hong DY (eds.) Flora of China, vol. 6. Science Press \& Missouri Botanical Garden, pp 333360

\section{Figures}




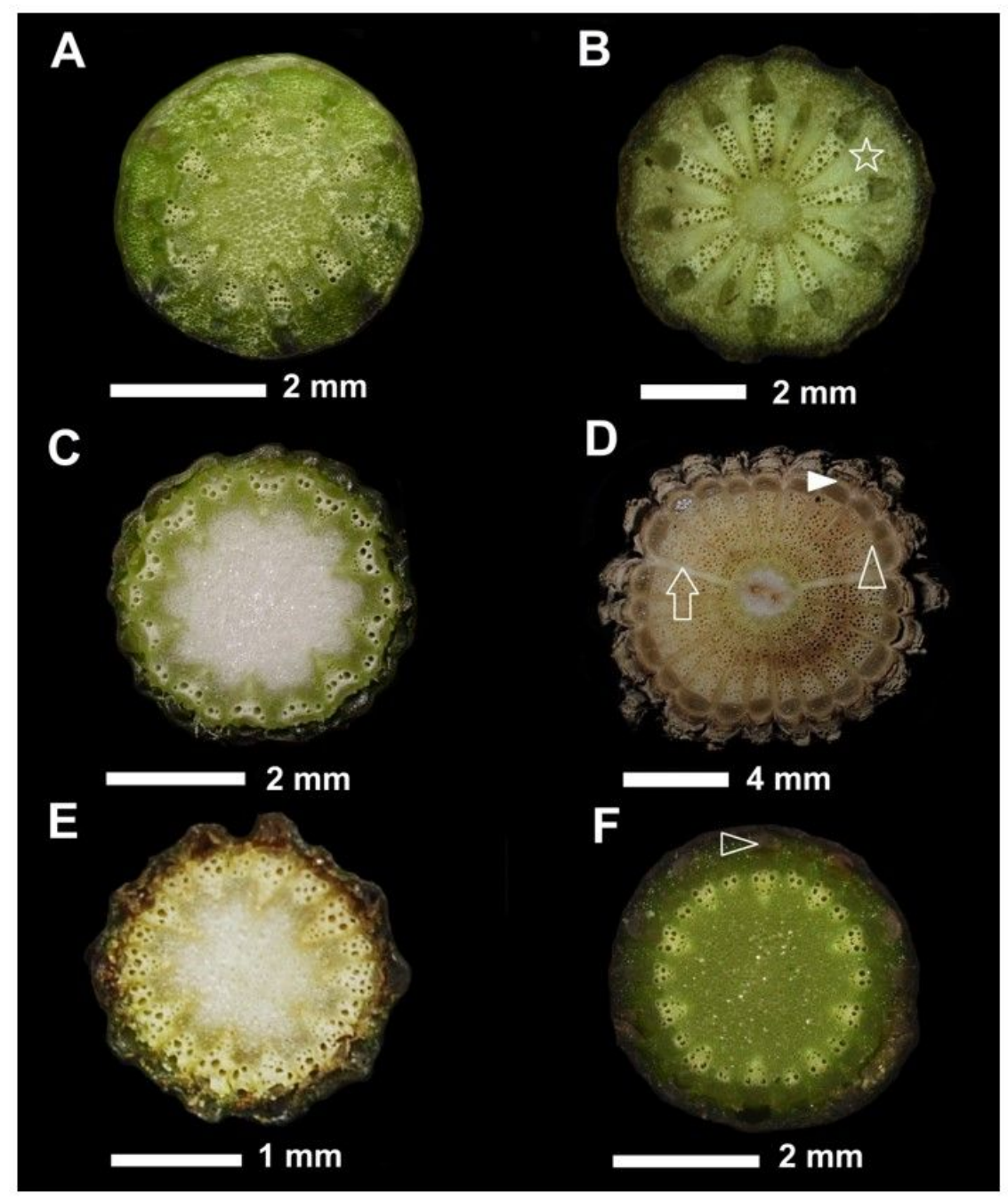

Figure 1

Stem transverse sections of Clematis species. A. C. akoensis; collenchyma strands and primary phloem fibers in dense bundles. B. C. akoensis; secondary phloem triangular in shape, wedge-like rays (open star). C. C. chinensis var. chinensis; phloem fiber strands, 10 big and 10 small vascular bundles. D. C. chinensis var. chinensis; cogwheel-like rhytidome with 3-4 layers, arc of bark (solid arrowhead), sequent periderm above semi-circular secondary phloem, 22 vascular bundles, 3 narrow rays (open arrowhead), two wide rays (open arrow). E. C. chinensis var. tatushanensis; stem with 10 shallow grooves, 10 big and 10 small vascular bundles. F. C. crassifolia; primary phloem fibers in dense bundles (open arrowhead), 10 big and 10 small vascular bundles. 


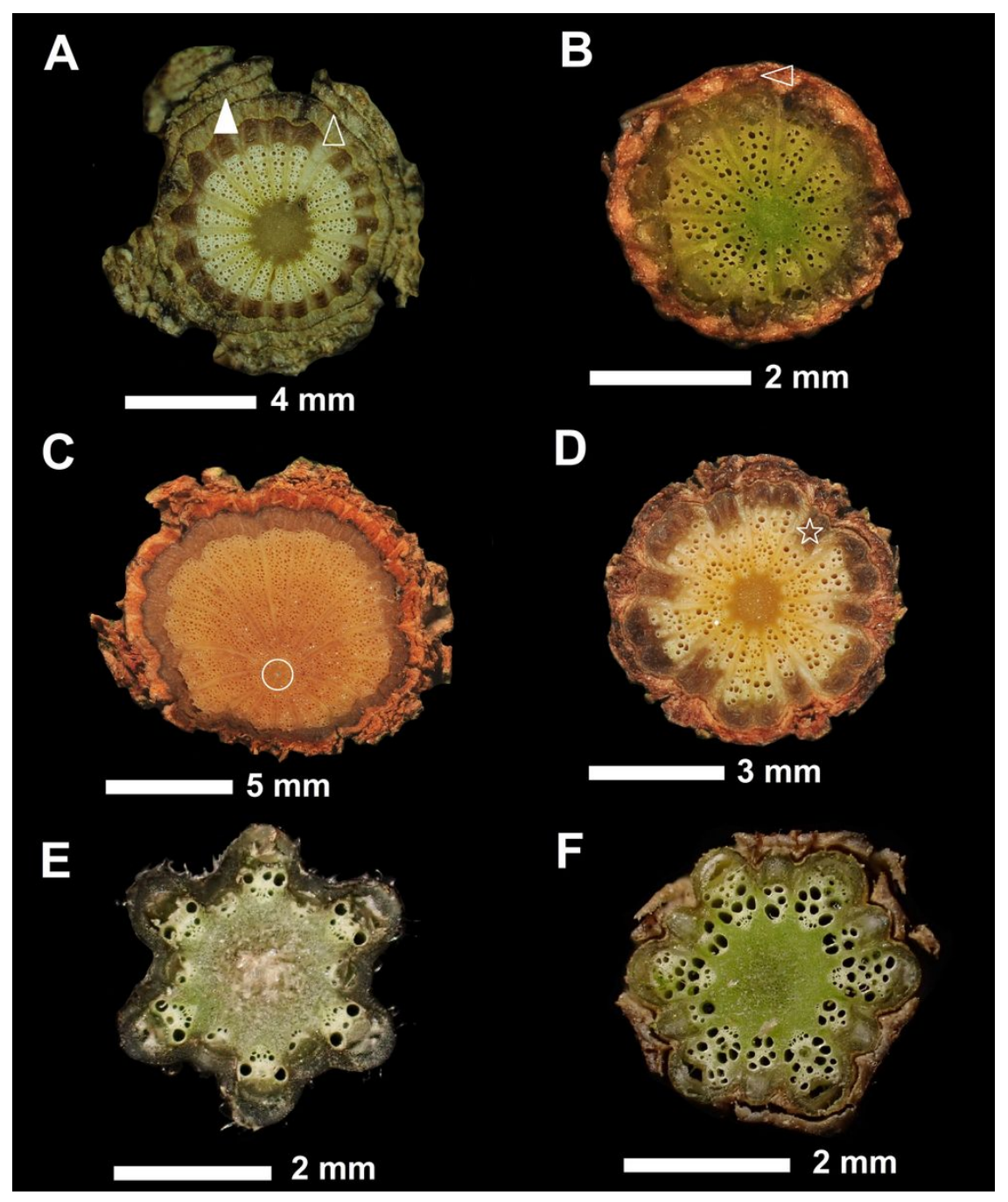

Figure 2

Stem transverse sections of Clematis species. A. C. crassifolia; sequent periderm produced in deeper layers within the secondary phloem, continuous segment rhytidome with 3-6 layers, each layer composed periderm (open arrowhead) and non-conducting phloem (solid arrowhead), ray dilatation, 6 wide rays. B. C. formosana; secondary phloem fiber strands (open arrowhead). C. C. formosana; secondary phloem fibers strands, black, continuous segment rhytidome with 2 layers, 32 narrow rays, eccentric pith (circle). D. C. gouriana subsp. lishanensis; ring-like sequent periderm, discontinuous wedge-like phloem (star), 12 wide rays, 14 narrow rays. E. C. grata; stem with 6 shallow grooves, phloem fiber in dense bundles beneath the angular extension of stem, 6 big and 6 small vascular bundles. F. C. grata; ring-like initial periderm, yellow, cogwheel-like rhytidome with 1 layer. 


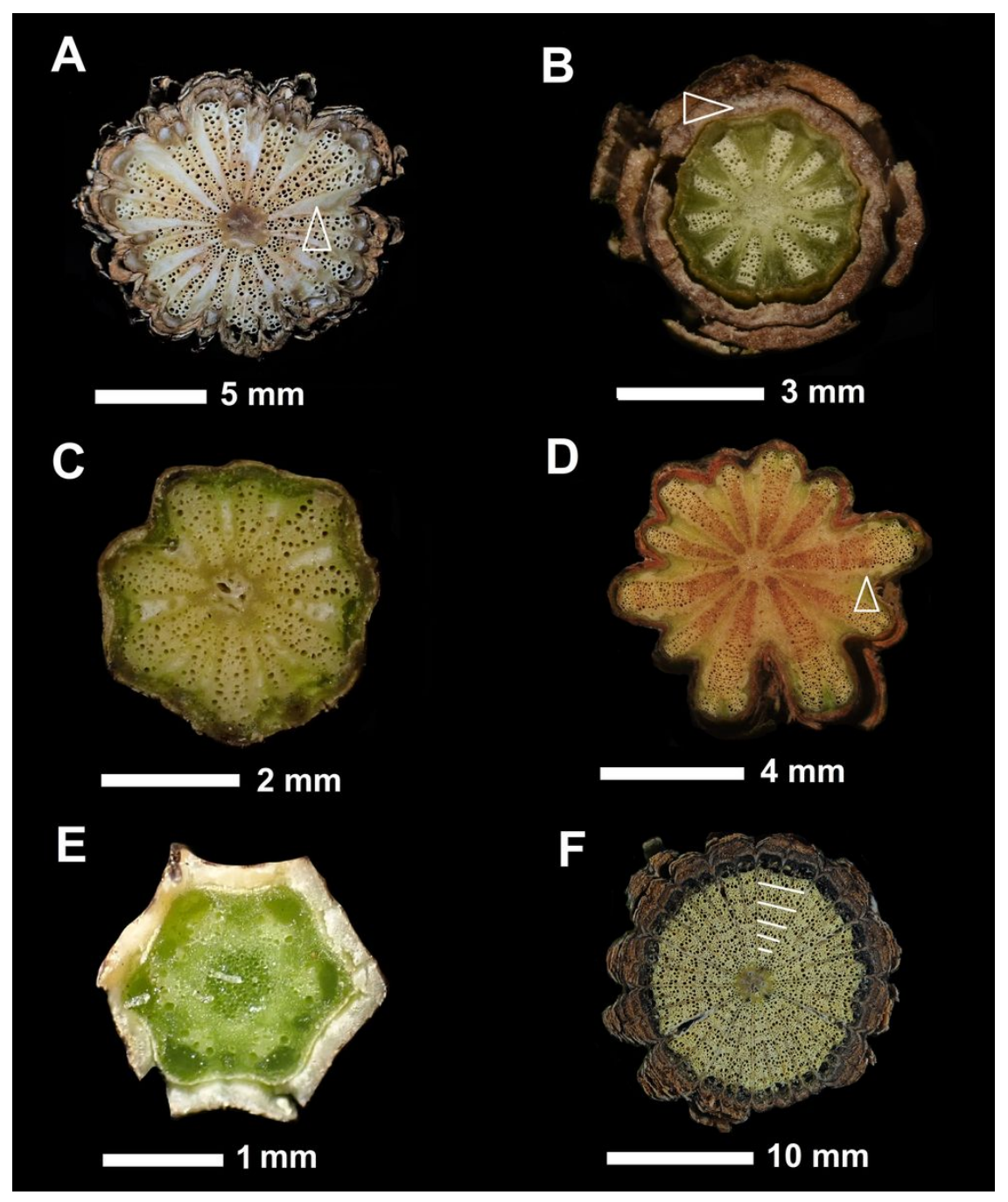

Figure 3

Stem transverse sections of Clematis species. A. C. grata; ray dilatation, wedge-like rays, 14 wide rays (open arrowhead), 11 narrow rays. B. C. henryi var. henryi; ring-like sequent periderm (open arrowhead), continuous segment rhytidome with 2 layers, wedge-like rays. C. C. henryi var. morii; stem shallowly lobed, wedge-like rays, ray dilatation, indentation near the wider rays, 10 vascular bundles. D. C. henryi var. morii; stem irregularly lobed, wedge-like rays, wedge-like indentation near the wider rays (open arrowhead), 11 vascular bundles. E. C. lasiandra; hexagon-shaped stem, 2 mm in diameter, 12 vascular bundles. F. C. lasiandra; arc-like bark, cogwheel-like rhytidome with 2-5 layers, 30 narrow rays, ring-porous vessels (lines). 


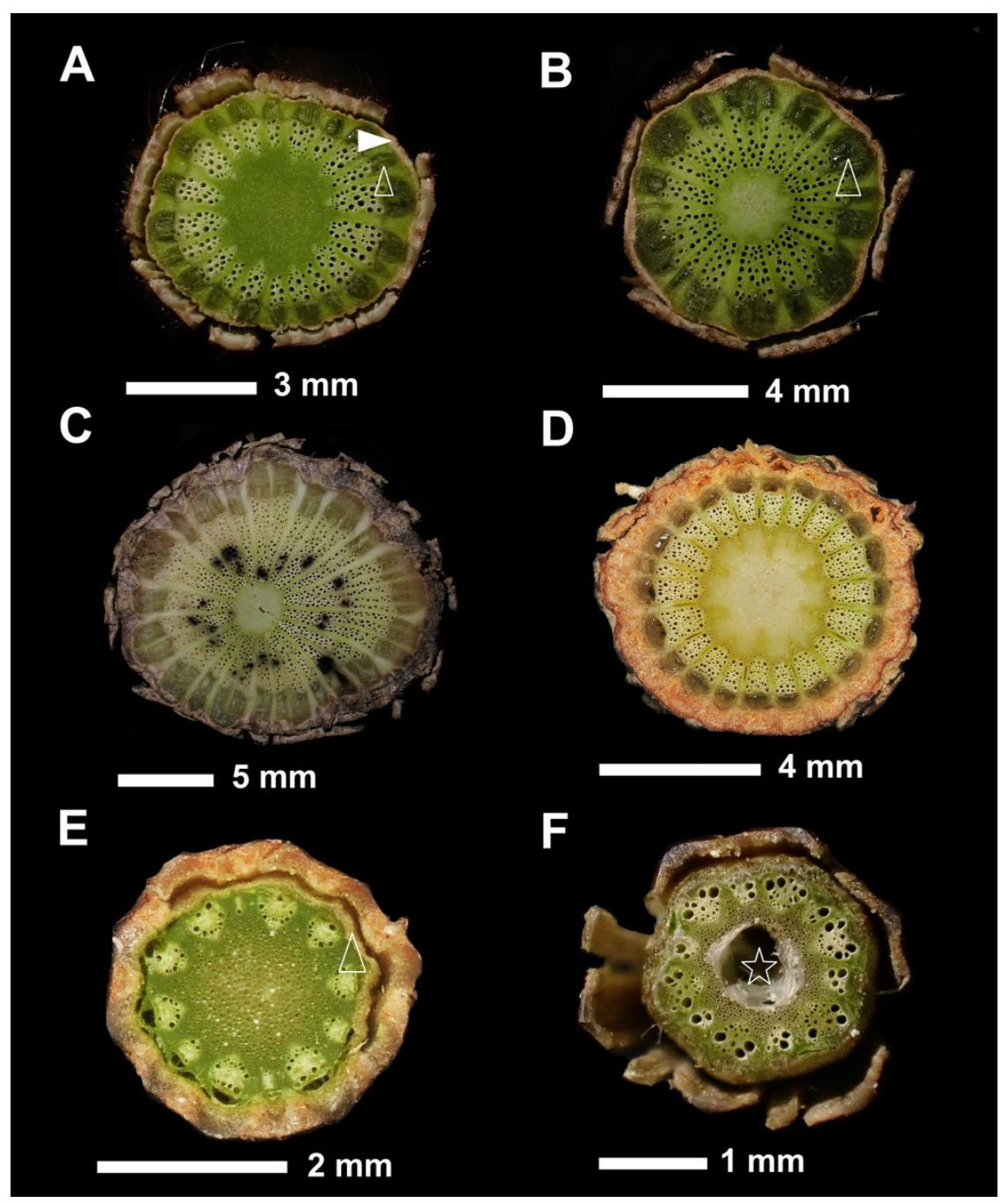

\section{Figure 4}

Stem transverse sections of Clematis species. A. C. leschenaultiana; semi-circular secondary phloem (open arrowhead), sequent periderm (solid arrowhead), continuous segment rhytidome with 2 layers, ray dilatation, 21 vascular bundles. B. C. leschenaultiana; aggregated secondary phloem (open arrowhead), 6 narrow rays, 16 vascular bundles. C. C. leschenaultiana; sequent periderms within the secondary phloem, continuous segment rhytidome with 4 layers, ray dilatation, 15 narrow rays, 19 vascular bundles. D. C. meyeniana; continuous segment rhytidome with 3 layer, sequent periderm within the secondary phloem, ray dilatation, indentation near the wider rays. E. C. montana; primary phloem fibers strands, ring-like initial periderm (open arrowhead), yellow. F. C. parviloba subsp. bartlettii; continuous segment rhytidome with 1 layer, 6 big and 6 small vascular bundles, pith cavity (open star). 


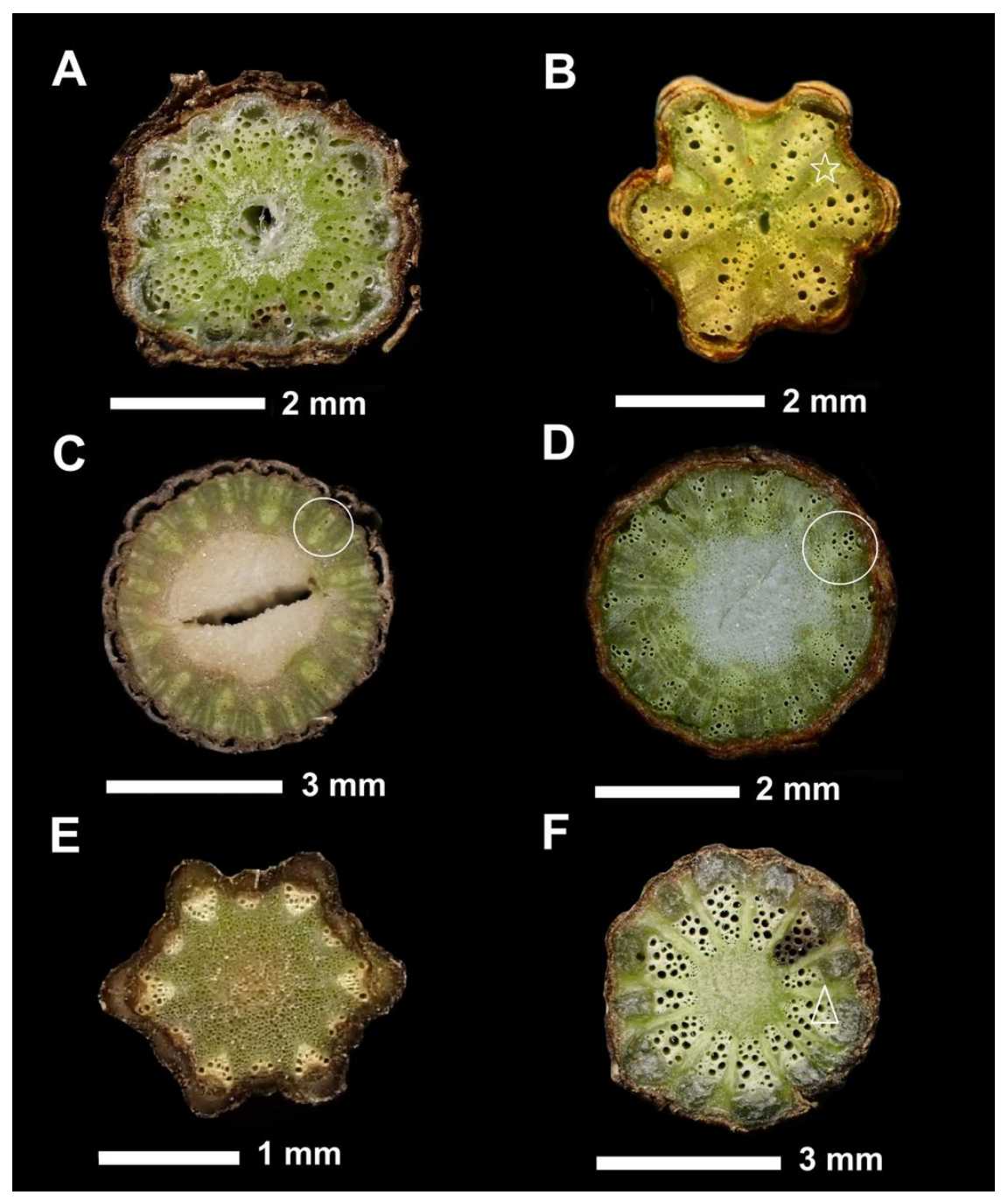

\section{Figure 5}

Stem transverse sections of Clematis species. A. C. parviloba subsp. bartlettii: ring-like sequent periderm, continuous segment rhytidome with 2 layers. B. C. psendotophora; stem with 6 shallow grooves, cogwheel-like rhytidome with 2 layers, wedge-like rays (open star), 6 vascular bundles. C-D. C. psilandra; vessel limited to the central portions of fascicular area (circle), 20-23 vessel bundles, pith cavity. E. C. tamurae; stem with 6 shallow grooves, primary phloem fiber ring-like, 6 big and 6 small vascular bundles. F. C. tamurae; secondary phloem dark-green and rectangular, ring-like sequent periderm, cogwheel-like rhytidome with 1 layer, ray dilatation, indentation of the axial parenchyma near the wider rays (open arrowhead). 


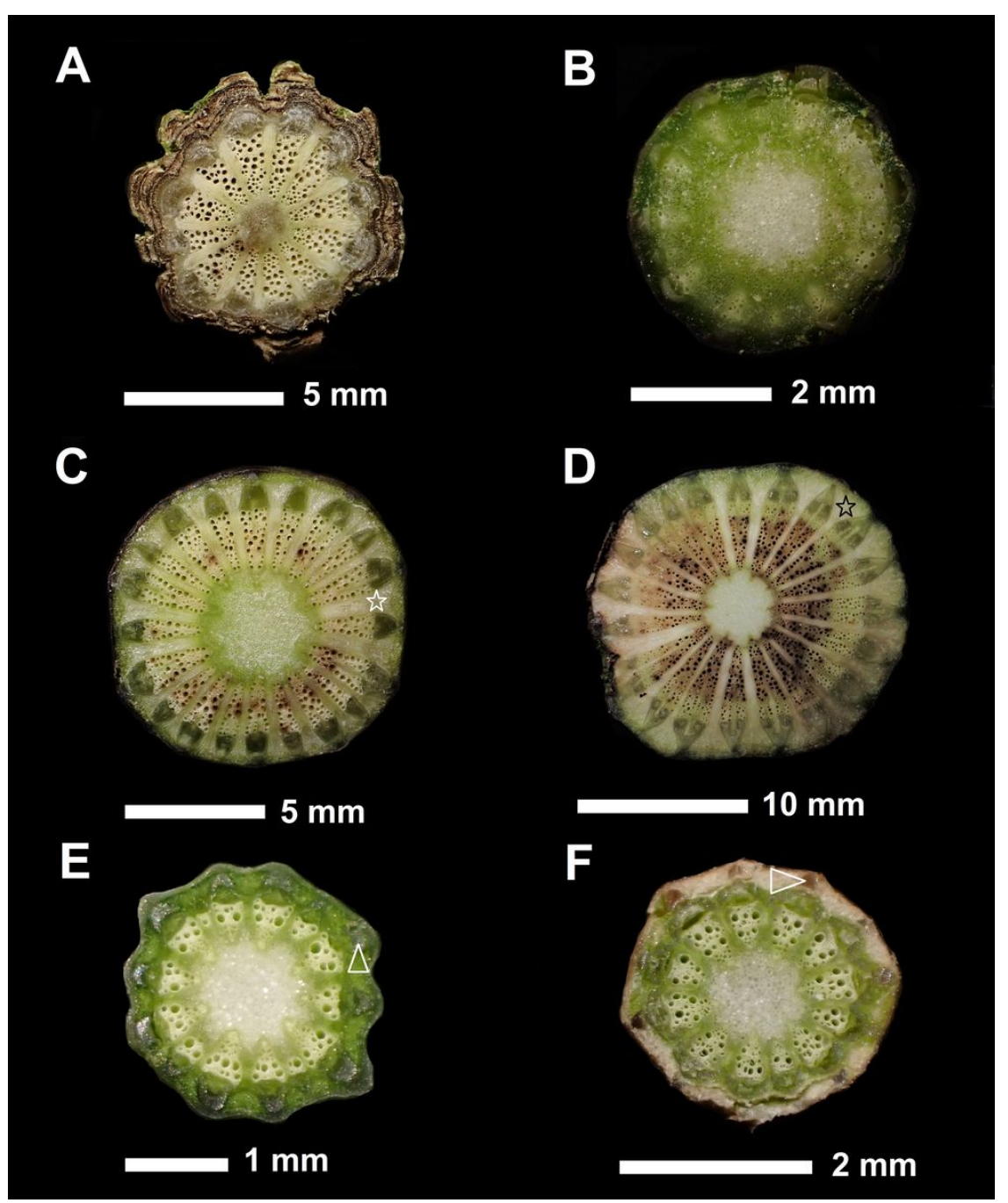

\section{Figure 6}

Stem transverse sections of Clematis species. A. C. tamurae; arc-like bark, sequent periderm within the secondary phloem, cogwheel-like rhytidome with 3-4 layers, ray dilatation, indentation near the wider rays. B. C. tashiroi; primary phloem fiber stands, 14 vascular bundles. C. C. tashiroi; secondary phloem triangular in shape and deep-green, ray dilatation, 4 wide rays (open star), 6 narrow rays. D. C. tashiroi; obvious ray dilatation (open star), 6 wide rays, 18 narrow rays. E. C. terniflora var. garanbiensis; collenchyma strands, primary phloem fibers bundles (open arrowhead), 14 vascular bundles. F. C. terniflora var. garanbiensis; primary phloem fibers triangular (open arrowhead), black, ring-like initial periderm. 


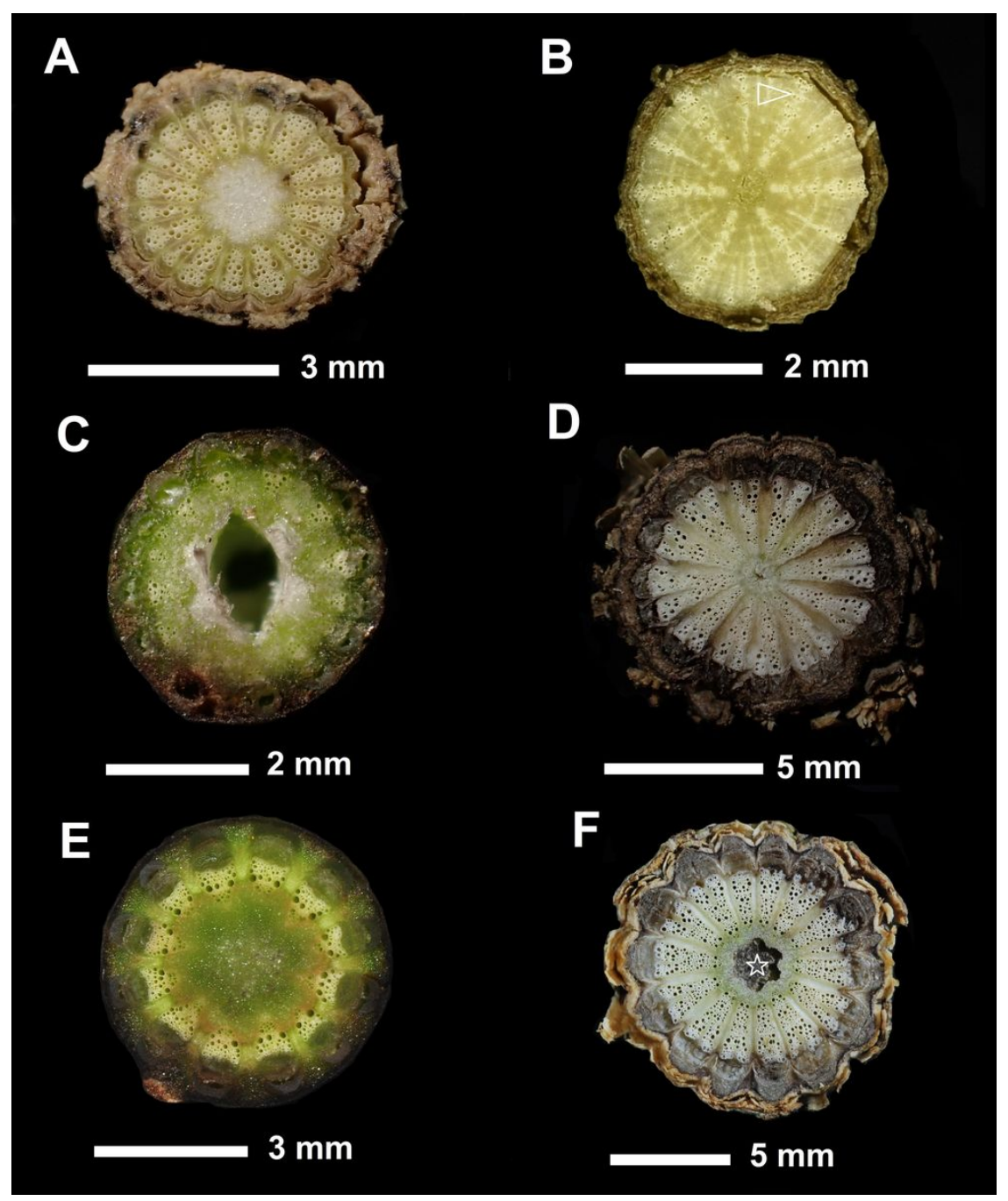

\section{Figure 7}

Stem transverse sections of Clematis species. A. C. terniflora var. garanbiensis; sequent periderm in the secondary phloem, cogwheel-like rhytidome with 2 layers. B. C. tsugetorum; vessels restricted to the central portions of fascicular area, vessel as growth rings (open arrowhead). C. C. uncinata var. okinawensis; primary phloem fibers strands, pith cavity. D. C. uncinata var. okinawensis; arc-like bark, cogwheel-like rhytidome with 1-3 layers, ray dilatation, 13 narrow rays. E. C. uncinata var. uncinata; primary phloem fibers strands, ray dilatation. F. C. uncinata var. uncinata; arc-like bark, 11 narrow rays, pith cavity, hexagon in shape (open star), primary xylem ring located around the pith. 